S. Niwa

Nagoya Math. J.

Vol. 121 (1991), 171-184

\title{
ON GENERALIZED WHITTAKER FUNCTIONS ON SIEGEL'S UPPER HALF SPACE OF DEGREE 2
}

\author{
S. NIWA
}

\section{Dedicated to Professor Tomio Kubota on his sixtieth birthday}

In [5], $\mathrm{H}$. Maass showed that the dimension of a space of generalized Whittaker functions satisfying certain system of differential equations on Siegel's upper half space $H_{2}$ of degree 2 is three. First of all, we shall investigate the structure of a space of generalized Whittaker functions which are eigen functions for the algebra of invariant differential operators on $H_{2}$. The theory of generalized Whittaker functions is discussed in Yamashita [12], [13], [14], [15] with full generality. But, we will get an outlook of the space of generalized Whittaker functions by using elementary calculus instead of representation theory of Lie groups. Generalized Whittaker functions, naturally appear in the theory of indefinite theta function, and so we shall next show commutation relations between the invariant differential operators on $H_{2}$ and those on the product $H_{1} \times H_{1}$ of two copies of the upper half plane $H_{1}$ operated on a theta function. The relations are analogies of commutation relations for Hecke operators in [1], [16], [17] and are proved in some cases with the Laplacian in [8], [2]. We essentially use the result in Nakajima [10] where the generators of the center of the universal enveloping algebra of $\mathfrak{s}_{\mathfrak{p}}(2, \mathbb{R})$ are explicitly given. By commutation relations we can construct an automorphic form $F$ on $H_{2}$ corresponding to an $L$-function with Grössencharacter of a certain biquadratic field. Generalized Whittaker functions investigated in the present paper appear in the Fourier expansion of $F$ with respect to translations in $\mathrm{H}_{2}$ and so we can define the "constant part" of the Fourier coefficient as the ratio of the Fourier coefficient to a generalized Whittaker function. The constant part of a certain Fourier coefficient of an automorphic form analogous to $F$ is given in 2. (See in particular (2.10).)

Received April 17, 1990. 
$\S 1$.

We denote, as usual, by $\mathbb{Z}, \mathbb{Q}, \mathbb{R}$, and $\mathbb{C}$ the ring of rational integers, the rational number field, the real number field, and the complex number field. We denote the algebra of invariant differential operators on $H_{2}$ by $\mathscr{D}$ and the center of the universal enveloping algebra of $\mathfrak{s p}(2, \mathbb{R})$ by $\mathscr{C}$. S. Nakajima calculated generators of $\mathscr{D}, \mathscr{C}$ in [9], [10]. The generators of $\mathscr{D}$ are

$$
\Delta_{1}=\sum_{i, j=1}^{3} y_{i} y_{j} \partial_{i} \bar{\partial}_{j}-d\left(\partial_{1} \bar{\partial}_{3}+\bar{\partial}_{1} \partial_{3}-(1 / 2) \partial_{2} \bar{\partial}_{2}\right)
$$

and

$$
\begin{aligned}
\Delta_{2}= & d^{2}\left(\partial_{1} \partial_{3}-(1 / 4) \partial_{2}^{2}\right)\left(\bar{\partial}_{1} \bar{\partial}_{3}-(1 / 4) \overline{\hat{\partial}}_{2}^{2}\right) \\
& +\sqrt{-1}(1 / 4) d\left(\sum_{i=1}^{3} y_{i} \partial_{i}\right)\left(\bar{\partial}_{1} \bar{\partial}_{3}-(1 / 4) \overline{\hat{o}}_{2}^{2}\right) \\
& +\sqrt{-1}(1 / 4) d\left(\sum_{i=1}^{3} y_{i} \bar{\partial}_{i}\right)\left(\partial_{1} \partial_{3}-(1 / 4) \partial_{2}^{2}\right) \\
& +(1 / 16) d\left(\partial_{1} \bar{\partial}_{3}+\bar{\partial}_{1} \partial_{3}-(1 / 2) \partial_{2} \bar{\partial}_{2}\right)
\end{aligned}
$$

where we put

$$
\begin{array}{ll}
z_{i}=x_{i}+\sqrt{-1} y_{i} \quad(1 \leq i \leq 3), \\
\partial_{i}=\frac{\partial}{\partial z_{i}}=(1 / 2)\left(\frac{\partial}{\partial x_{i}}-\sqrt{-1} \frac{\partial}{\partial y_{i}}\right) \quad(1 \leq i \leq 3), \\
\bar{\partial}_{i}=\frac{\partial}{\partial \bar{z}_{i}}=(1 / 2)\left(\frac{\partial}{\partial x_{i}}+\sqrt{-1} \frac{\partial}{\partial y_{i}}\right) \quad(1 \leq i \leq 3),
\end{array}
$$

and $d=y_{1} y_{3}-y_{2}^{2}$ for $\left(\begin{array}{ll}z_{1} & z_{2} \\ z_{2} & z_{3}\end{array}\right) \in H_{2}$. For two complex numbers $d_{1}, d_{2}$, we consider the space $\mathscr{W}$ formed by functions $f(Z)=g(Y) e^{2 \pi i \operatorname{tr} X}$ of $Z=X+$ $i Y \in H_{2}$, satisfying $\Delta_{1} f=d_{1} f$ and $\Delta_{2} f=d_{2} f$ with some functions $g$ of $Y$, which we call generalized Whittaker functions. If $g(Y) e^{2 \pi i \operatorname{tr} X}$ belongs to $\mathscr{W}, g$ has to satisfy

$$
\begin{aligned}
& \frac{1}{8}\left(4 y_{3} y_{2} \frac{\partial^{2}}{\partial y_{3} \partial y_{2}}+4 y_{2}^{2} \frac{\partial^{2}}{\partial y_{3} \partial y_{1}}+2 y_{3}^{2} \frac{\partial^{2}}{\partial y_{3}^{2}}+4 y_{2} y_{1} \frac{\partial^{2}}{\partial y_{2} \partial y_{1}}\right. \\
& \left.+y_{3} y_{1} \frac{\partial^{2}}{\partial y_{2}^{2}}+y_{2}^{2} \frac{\partial^{2}}{\partial y_{2}^{2}}+2 y_{1}^{2} \frac{\partial^{2}}{\partial y_{1}^{2}}-8 \pi^{2} y_{3}^{2}-16 \pi^{2} y_{2}^{2}-8 \pi^{2} y_{1}^{2}\right) g=d_{1} g
\end{aligned}
$$

and 


$$
\begin{aligned}
& -\frac{d}{256}\left(\left(2\left(16 d \pi^{2}+1\right) \frac{\partial^{2}}{\partial y_{2}^{2}}-32\left(8 d \pi^{2}-1\right) \pi^{2}\right.\right. \\
& \quad+d\left(8 \frac{\partial^{4}}{\partial y_{1} \partial y_{2}^{2} \partial y_{3}}-16 \frac{\partial^{4}}{\partial y_{1}^{2} \partial y_{3}^{2}}+64 \pi^{2} \frac{\hat{o}^{2}}{\partial y_{1}^{2}}-\frac{\partial^{4}}{\partial y_{2}^{4}}+64 \pi^{2} \frac{\partial^{2}}{\partial y_{3}^{2}}\right) \\
& \quad+16 y_{2} \frac{\partial^{3}}{\partial y_{1} \partial y_{2} \partial y_{3}}-4 y_{1} \frac{\partial^{3}}{\partial y_{1} \partial y_{2}^{2}}+16 y_{3} \frac{\partial^{3}}{\partial y_{1} \partial y_{3}^{2}}-8 \frac{\partial^{2}}{\partial y_{1} \partial y_{3}} \\
& \quad+16 y_{1} \frac{\partial^{3}}{\partial y_{1}^{2} \partial y_{3}}-64 \pi^{2} y_{3} \frac{\partial}{\partial y_{1}}-4 y_{2} \frac{\partial^{3}}{\partial y_{2}^{3}}-4 y_{3} \frac{\partial^{3}}{\partial y_{2}^{2} \partial y_{3}} \\
& \left.\quad+64 \pi^{2} y_{2} \frac{\partial}{\partial y_{2}}-64 \pi^{2} y_{1} \frac{\partial}{\partial y_{1}}\right) g=d_{2} g
\end{aligned}
$$

with $d=y_{1} y_{3}-y_{2}^{2}$. Put

$$
Y=\left(\begin{array}{ll}
y_{1} & y_{2} \\
y_{2} & y_{3}
\end{array}\right)=\left(\begin{array}{ll}
t_{1} & 0 \\
0 & t_{2}
\end{array}\right)\left[\begin{array}{rr}
\cos \theta & -\sin \theta \\
\sin \theta & \cos \theta
\end{array}\right], \quad g(Y)=h\left(t_{1}, t_{2}, \theta\right) .
$$

Then $h$ is periodic with respect to $\theta$, so that $h$ has a Fourier expansion

$$
h\left(t_{1}, t_{2}, \theta\right)=\sum_{n \in \mathbf{Z}} B_{n}\left(t_{1}, t_{2}\right) e^{2 n i \theta}
$$

where $B_{n}\left(t_{1}, t_{2}\right)$ is a solution of differential equations

$$
\begin{aligned}
& -4^{-1}\left(t_{1}-t_{2}\right)^{-2}\left(4\left(t_{1}^{2}+t_{2}^{2}\right)\left(t_{1}-t_{2}\right)^{2} \pi^{2}+2 n^{2} t_{1} t_{2}\right. \\
& \left.-\left(t_{1}-t_{2}\right)^{2}\left(t_{1}^{2} \frac{\partial^{2}}{\partial t_{1}^{2}}+t_{2}^{2} \frac{\partial^{2}}{\partial t_{2}^{2}}\right)-t_{1} t_{2}\left(t_{1}-t_{2}\right)\left(\frac{\partial}{\partial t_{1}}-\frac{\partial}{\partial t_{2}}\right)\right) B_{n}=d_{1} B_{n}
\end{aligned}
$$

and

$$
\begin{aligned}
& \frac{t_{1} t_{2}}{64\left(t_{1}-t_{2}\right)^{4}}\left(2\left(16\left(t_{1}-t_{2}\right)^{2} \pi^{2} t_{1} t_{2}+5 t_{1}^{2}-18 t_{1} t_{2}+5 t_{2}^{2}\right) n^{2}\right. \\
& \quad+8\left(8 \pi^{2} t_{1} t_{2}-1\right)\left(t_{1}-t_{2}\right)^{4} \pi^{2}+4 n^{4} t_{1} t_{2} \\
& \quad-\left(t_{1}-t_{2}\right)\left(4\left(2 t_{1}-t_{2}\right) n^{2} t_{2}-16\left(t_{1}-t_{2}\right)^{2} \pi^{2} t_{1}^{2}-3 t_{1}^{2}+8 t_{1} t_{2}-3 t_{2}^{2}\right) \frac{\partial}{\partial t_{2}} \\
& \quad-\left(t_{1}-t_{2}\right)^{2} t_{2}\left(16\left(t_{1}-t_{2}\right)^{2} \pi^{2} t_{1}+3 t_{1}-2 t_{2}\right) \frac{\partial^{2}}{\partial t_{2}^{2}} \\
& \quad-\left(t_{1}-t_{2}\right)\left(16\left(t_{1}-t_{2}\right)^{2} \pi^{2} t_{2}^{2}+4\left(t_{1}-2 t_{2}\right) n^{2} t_{1}+3 t_{1}^{2}-8 t_{1} t_{2}+3 t_{2}^{2}\right) \frac{\partial}{\partial t_{1}} \\
& \quad-t_{1}\left(t_{1}-t_{2}\right)^{2}\left(16\left(t_{1}-t_{2}\right)^{2} \pi^{2} t_{2}^{2}-2 t_{1}+3 t_{2}\right) \frac{\partial^{2}}{\partial t_{1}^{2}} \\
& \quad+4 t_{2}^{2}\left(t_{1}-t_{2}\right)^{3} \frac{\partial^{3}}{\partial t_{1} \partial t_{2}^{2}}-4 t_{1}^{2}\left(t_{1}-t_{2}\right)^{3} \frac{\partial^{3}}{\partial t_{1}^{2} \partial t_{2}} \\
& \left.\quad+2\left(4 n^{2}+1\right) t_{1} t_{2}\left(t_{1}-t_{2}\right)^{2} \frac{\partial^{2}}{\partial t_{1} \partial t_{2}}+4 t_{1} t_{2}\left(t_{1}-t_{2}\right)^{4} \frac{\partial^{4}}{\partial t_{1}^{2} \partial t_{2}^{2}}\right) B_{n}=d_{2} B_{n} .
\end{aligned}
$$


Since the differential equations do not change when $n \rightarrow-n$, we assume $n \geq 0$ hereafter. Now we introduce variables $x=t_{1}-t_{2}, y=t_{1}+t_{2}$ and put

$$
B_{n}\left(t_{1}, t_{2}\right)=C_{n}(x, y)
$$

where $y>0,-y<x<y$. Then the differential equations for $B_{n}$ yield the following condition for $C_{n}$ :

$$
\begin{aligned}
& -\frac{1}{8 x^{2}}\left(4 \pi^{2} x^{2}\left(x^{2}+y^{2}\right)+\left(y^{2}-x^{2}\right) n^{2}-x^{2}\left(x^{2}+y^{2}\right) \frac{\partial^{2}}{\partial y^{2}}\right. \\
& \left.-x^{2}\left(x^{2}+y^{2}\right) \frac{\partial^{2}}{\partial x^{2}}-x\left(y^{2}-x^{2}\right) \frac{\partial}{\partial x}-4 x^{3} y \frac{\partial^{2}}{\partial x \partial y}\right) C_{n}=d_{1} C_{n} \\
& \frac{\left(y^{2}-x^{2}\right)}{256 x^{4}}\left(\left(n^{4}+16 \pi^{4} x^{4}\right)\left(y^{2}-x^{2}\right)+2\left(4 \pi^{2} x^{2} y^{2}-4 \pi^{2} x^{4}-2 y^{2}+7 x^{2}\right) n^{2}\right. \\
& \quad-8 \pi^{2} x^{4}+4 x^{3} y\left(x \frac{\partial^{3}}{\partial y \partial x^{2}}+\frac{\partial^{2}}{\partial x \partial y}-x \frac{\partial^{3}}{\partial y^{3}}\right) \\
& \quad+x^{4}\left(y^{2}-x^{2}\right)\left(\frac{\partial^{4}}{\partial y^{4}}-2 \frac{\partial^{4}}{\partial x^{2} \partial y^{2}}+\frac{\partial^{4}}{\partial x^{4}}\right) \\
& \quad-2 x^{3}\left(y^{2}+x^{2}\right)\left(\frac{\partial^{3}}{\partial x \partial y^{2}}-\frac{\partial^{3}}{\partial x^{3}}\right) \\
& \quad-x^{2}\left(2 n^{2} y^{2}-2 n^{2} x^{2}+8 \pi^{2} y^{2} x^{2}-8 \pi^{2} x^{4}+y^{2}-3 x^{2}\right) \frac{\partial^{2}}{\partial x^{2}} \\
& \quad+x\left(2 n^{2} y^{2}-6 n^{2} x^{2}-8 \pi^{2} x^{2} y^{2}-8 \pi^{2} x^{4}+y^{2}-7 x^{2}\right) \frac{\partial}{\partial x} \\
& \quad+2 x^{2}\left(n^{2} y^{2}-n^{2} x^{2}-4 \pi^{2} x^{2} y^{2}+4 \pi^{2} x^{4}+x^{2}\right) \frac{\partial^{2}}{\partial y^{2}} \\
& \left.\quad-4 x^{2} y\left(n^{2}+4 \pi^{2} x^{2}\right) \frac{\partial}{\partial y}\right) C_{n}=d_{2} C_{n} .
\end{aligned}
$$

Put $C_{n}(x, y)=\left(x^{2}-y^{2}\right) \sum_{k=0}^{\infty} a_{k}(y) x^{k}$ where $a_{k}(y)=0$ if $k \leq m$ with an integer $m \geq 0$ such that $a_{m}(y) \neq 0$. Then the equation (1.8.1) implies

$$
\begin{gathered}
\frac{1}{8}\left(\left(k^{2}+2 k+n^{2}-4 \pi^{2} y^{2}-2\right) a_{k}+\left((k+2)^{2}-n^{2}\right) y^{2} a_{k+2}-4 \pi^{2} a_{k-2}\right. \\
\left.+a_{k-2}^{\prime \prime}+y^{2} a_{k}^{\prime \prime}+(4 k+4) y a_{k}^{\prime}\right)=d_{1} a_{k}
\end{gathered}
$$

especially $\left(m^{2}-n^{2}\right) y^{2} a_{m}=0, \quad\left((m+1)^{2}-n^{2}\right) y^{2} a_{m+1}=0$ which results in $m=n, a_{m+1}=0$ and therefore $a_{k}=0$ unless $k \equiv m \bmod 2$. The equation (1.8.2) implies 


$$
\begin{aligned}
- & \frac{1}{256}\left(2(k+n+2)(k-n+2) y^{3}\left(y a_{k+2}^{\prime \prime}+2 a_{k+2}^{\prime}\right)\right. \\
& -\left(k^{4}-2 k^{2} n^{2}+16 k^{2} \pi^{2} y^{2}-6 k^{2}+16 k \pi^{2} y^{2}\right. \\
& \left.-16 k+n^{4}-16 n^{2} \pi^{2} y^{2}-10 n^{2}+16 \pi^{4} y^{4}+40 \pi^{2} y^{2}+12\right) a_{k} \\
& -2\left(2 k^{2}+2 k-2 n^{2}-4 \pi^{2} y^{2}-1\right) y^{2} a_{k}^{\prime \prime} \\
& +2\left(k^{2}+2 k-n^{2}+4 \pi^{2} y^{2}+7\right)(k+n+2)(k-n+2) y^{2} a_{k+2} \\
& +8\left(k^{2}-2 k-n^{2}+4 \pi^{2} y^{2}-3\right) \pi^{2} a_{k-2} \\
& +2\left(k^{2}-2 k-n^{2}-8 \pi^{2} y^{2}+7\right) a_{k-2}^{\prime \prime} \\
& -4\left(k^{2}-8 k-n^{2}-4 \pi^{2} y^{2}-8\right) y a_{k}^{\prime} \\
& -(k+n+4)(k+n+2)(k-n+4)(k-n+2) y^{4} a_{k+4} \\
& -a_{k-4}^{(4)}+8 \pi^{2} a_{k-4}^{\prime \prime}+2 y^{2} a_{k-2}^{(4)}+4 y a_{k-2}^{(3)}-16 \pi^{2} y a_{k-2}^{\prime} \\
& \left.-y^{4} a_{k}^{(4)}-4 y^{3} a_{k}^{(3)}-16 \pi^{4} a_{k-4}\right)=d_{2} a_{k}
\end{aligned}
$$

for all integers $k$. Since we especially have

$$
\begin{aligned}
& a_{n+2}=\frac{1}{4(n+1) y^{2}}\left(2\left(4 d_{1}-n^{2}-n+2 \pi^{2} y^{2}+1\right) a_{n}\right.\left.-4(n+1) y a_{n}^{\prime}-y^{2} a_{n}^{\prime \prime}\right), \\
& a_{n+4}=\frac{1}{8(n+2) y^{2}}\left(2\left(4 d_{1}-n^{2}-3 n+2 \pi^{2} y^{2}-3\right) a_{n+2}\right. \\
&\left.\quad-4(n+3) y a_{n+2}^{\prime}-y^{2} a_{n+2}^{\prime \prime}-a_{n}^{\prime \prime}+4 \pi^{2} a_{n}\right)
\end{aligned}
$$

and

$$
\begin{aligned}
& 4\left(64 d_{2}+4 n^{2}-4 n \pi^{2} y^{2}+4 n-4 \pi^{4} y^{4}-10 \pi^{2} y^{2}-3\right) a_{n} \\
& \quad+8 y^{2}\left(2 n+4 \pi^{2} y^{2}+7\right)(n+1) a_{n+2}+16\left(2 n+\pi^{2} y^{2}+2\right) y a_{n} \\
& \quad-2\left(2 n-4 \pi^{2} y^{2}-1\right) y^{2} a_{n}^{\prime \prime}-32(n+2)(n+1) a_{n+4} y^{4} \\
& +8 y^{3}(n+1)\left(y a_{n+2}^{\prime \prime}+2 a_{n+2}^{\prime}\right)-y^{4} a_{n}^{(4)}-4 y^{3} a_{n}^{(3)}=0,
\end{aligned}
$$

we obtain from (1.9.1), (1.9.2) an ordinary differential equation

$$
\begin{aligned}
\left(8 \left(n^{2}+\right.\right. & \left.+n+1) d_{1}-16 d_{1}^{2}+64 d_{2}-n^{4}-2 n^{3}+n^{2}+2 n+8 \pi^{2} y^{2}\right) a_{n} \\
& +4\left(4(n+1) d_{1}-n^{3}-3 n^{2}-2 n+4 \pi^{2} y^{2}\right) y a_{n}^{\prime} \\
& +2\left(4 d_{1}-3 n^{2}-9 n+2 \pi^{2} y^{2}-6\right) y^{2} a_{n}^{\prime \prime} \\
& -4(n+2) y^{3} a_{n}^{(3)}-y^{4} a_{n}^{(4)}=0
\end{aligned}
$$

for $a_{n}$. It is more convenient to introduce parameters $\lambda_{1}, \lambda_{2}$ defined by

$$
d_{1}=\frac{\lambda_{1}+\lambda_{2}-2}{8}, \quad d_{2}=\frac{\left(\lambda_{1}-\lambda_{2}\right)^{2}}{256}-\frac{\lambda_{1}+\lambda_{2}}{32}+\frac{3}{64}
$$

to describe the solutions of (1.12). With these $\lambda_{1}, \lambda_{2}$ (1.12) becomes 


$$
\begin{aligned}
& -\left(\lambda_{1} \lambda_{2}-n(n+1) \lambda_{1}-n(n+1) \lambda_{2}+n^{4}+2 n^{3}+n^{2}-8 \pi^{2} y^{2}\right) a_{n} \\
& \quad+2\left(\left(\lambda_{1}+\lambda_{2}\right)(n+1)-2 n^{3}-6 n^{2}-6 n+8 \pi^{2} y^{2}-2\right) y a_{n}^{\prime} \\
& \quad+\left(\lambda_{1}+\lambda_{2}-6 n^{2}-18 n+4 \pi^{2} y^{2}-14\right) y^{2} a_{n}^{\prime \prime} \\
& \quad-4(n+2) y^{3} a_{n}^{(3)}-y^{4} a_{n}^{(4)}=0 .
\end{aligned}
$$

For $\nu \in \mathbb{C}, m \in \mathbb{Z}$, denote the associated Legendre function of the first kind by $P_{\nu}^{m}(z)$ and that of the second kind by $Q_{\nu}^{m}(z)$ as usual. Then we have

$$
\begin{aligned}
& P_{\nu}^{m}(z)=\frac{\Gamma(\nu+m+1)}{\pi \Gamma(\nu+1)} \int_{0}^{\pi}\left(z+\left(z^{2}-1\right)^{1 / 2} \cos t\right)^{\nu} \cos m t d t, \\
& Q_{\nu}^{m}(z)=(-1)^{m} \frac{\Gamma(\nu+m+1)}{2^{\nu+1} \Gamma(\nu+1)}\left(z^{2}-1\right)^{m / 2} \int_{-1}^{1} \frac{\left(1-t^{2}\right)^{\nu}}{(z-t)^{\nu+m+1}} d t
\end{aligned}
$$

for $z$ not on the real axis between 1 and $-\infty$, assuming $w^{\mu}=e^{\mu \log w}$, $\log w=\log |w|+i \arg w,-\pi<\arg w<\pi$ for $w, \mu \in \mathbb{C}$. We put

$$
\begin{aligned}
& P_{\nu}^{m}(x)=\lim _{y \rightarrow+0} e^{3 m_{\pi} i / 2} P_{\nu}^{m}(x+i y)=\lim _{y \rightarrow+0} e^{m_{\pi i / 2}} P_{\nu}^{m}(x-i y), \\
& Q_{\nu}^{m}(x)=\frac{1}{2} \lim _{y \rightarrow+0}\left(e^{-m_{\pi} i / 2} Q_{\nu}^{m}(x+i y)-e^{m_{\pi i / 2}} Q_{\nu}^{m}(x-i y)\right)
\end{aligned}
$$

for $-1<x<1 . \quad P_{\nu}^{m}(z)$ and $Q_{\nu}^{m}(z)$ are independent solutions of Legendre's differential equation

$$
\frac{d}{d z}\left(\left(1-z^{2}\right) \frac{d}{d z}\right) u+\nu(\nu+1) u-\frac{m^{2}}{1-z^{2}} u=0 .
$$

Put

$$
\begin{aligned}
& c_{11}=-Q_{\nu_{2}}^{0}(0)=\sqrt{\pi} 2^{-1} \sin \left(\frac{\nu_{2}}{2} \pi\right) \Gamma\left(\frac{\nu_{2}+1}{2}\right) / \Gamma\left(\frac{\nu_{2}}{2}+1\right), \\
& c_{12}=P_{\nu_{2}}^{0}(0)=\sqrt{\pi} /\left(\Gamma\left(\frac{\nu_{2}}{2}+1\right) \Gamma\left(\frac{-\nu_{2}+1}{2}\right)\right), \\
& c_{21}=-\frac{d}{d z} Q_{\nu_{2}}^{0}(0)=-\sqrt{\pi} \cos \left(\frac{\nu_{2}}{2} \pi\right) \Gamma\left(\frac{\nu_{2}}{2}+1\right) / \Gamma\left(\frac{\nu_{2}+1}{2}\right), \\
& c_{22}=\frac{d}{d z} P_{\nu_{2}}^{0}(0)=2 \sin \left(\frac{\nu_{2}}{2} \pi\right) \Gamma\left(\frac{\nu_{2}}{2}+1\right) /\left(\sqrt{\pi} \Gamma\left(\frac{\nu_{2}+1}{2}\right)\right) .
\end{aligned}
$$

Then $c_{12} \neq 0, c_{22} \neq 0$ for $-1<\Re e \nu_{2}<0$. Put

$$
\begin{aligned}
& R_{\nu_{2}}^{0}(z)=c_{11} P_{\nu_{2}}^{0}(z)+c_{12} Q_{\nu_{2}}^{0}(z), \\
& S_{\nu_{2}}^{0}(z)=c_{21} P_{\nu_{2}}^{0}(z)+c_{22} Q_{\nu_{2}}^{0}(z) .
\end{aligned}
$$

Then we obtain 
Proposition 1. Put $\nu_{1}=\frac{-1+\sqrt{1+4 \lambda_{1}}}{2}, \nu_{2}=\frac{-1+\sqrt{1+4 \lambda_{2}}}{2}$, and assume that $-1<\Re e \nu_{1}<0,-1<\Re e \nu_{2}<0$ and that $\lambda_{1}, \lambda_{2}$ are not integers. Then there exist polynomials $h_{1}, h_{2}$ in $y^{-1}$ of degree $n-1, n$ such that

$$
\begin{aligned}
& A_{n}(y)=\int_{0}^{\infty}\left(\int_{1}^{\infty} R_{\nu_{1}}^{0}\left(z_{1}\right) P_{\nu_{2}}^{n}\left(z_{2}\right)\left(-2 \pi y z_{2}\right)^{-n}\left(z_{2}^{2}-1\right)^{n / 2} e^{-2 \pi z_{1} z_{2} y} d z_{2}\right) d z_{1}-h_{1}, \\
& B_{n}(y)=\int_{0}^{\infty}\left(\int_{1}^{\infty} S_{\nu_{1}}^{0}\left(z_{1}\right) P_{\nu_{2}}^{n}\left(z_{2}\right)\left(-2 \pi y z_{2}\right)^{-n}\left(z_{2}^{2}-1\right)^{n / 2} e^{-2 \pi z_{1} z_{2} y} d z_{2}\right) d z_{1}-h_{2} \\
& C_{n}(y)=\int_{1}^{\infty} \int_{1}^{\infty} P_{\nu_{1}}^{n}\left(z_{1}\right) P_{\nu_{2}}^{n}\left(z_{2}\right)\left(z_{1}^{2}-1\right)^{n / 2}\left(z_{2}^{2}-1\right)^{n / 2} e^{-2 \pi z_{1} z_{2} y} d z_{1} d z_{2}
\end{aligned}
$$

are linearly independent solutions of the equation (1.14). Further assume that $\lambda_{1}, \lambda_{2}$ are real. Then there exists a polynomial $h_{3}$ in $y^{-1}$ of degree $n$ such that together with above three functions

$$
\begin{aligned}
D_{n}(y)=\int_{0}^{1}\left(\int_{-1}^{1} P_{\nu_{1}}^{n}\left(z_{1}\right) P_{\nu_{2}}^{n}\left(-z_{2}\right)\left(z_{1}^{2}-1\right)^{n / 2}\left(z_{2}^{2}-1\right)^{n / 2} e^{-2 \pi z_{1} z_{2} y} d z_{2}\right) d z_{1} \\
+\sqrt{-1} \frac{\Gamma\left(\nu_{1}+n+1\right)}{\Gamma\left(\nu_{1}-n+1\right)} \int_{0}^{1}\left(\int_{1}^{\infty} P_{\nu_{1}}^{0}\left(z_{1}\right) P_{\nu_{2}}^{n}\left(-z_{2}\right)\left(-2 \pi y z_{2}\right)^{-n}\right. \\
\left.\times\left(z_{2}^{2}-1\right)^{n / 2} e^{-2 \pi z_{1} z_{2} y} d z_{2}\right) d z_{1}+h_{3}
\end{aligned}
$$

generates all the solutions of the equation (1.14).

Note that polynomials $h_{1}, h_{2}, h_{3}$ can be given explicitly.

Proof. Put

$$
\begin{aligned}
L= & -\left(\lambda_{1} \lambda_{2}-n(n+1) \lambda_{1}-n(n+1) \lambda_{2}+n^{4}+2 n^{3}+n^{2}-8 \pi^{2} y^{2}\right) \\
& +2\left(\left(\lambda_{1}+\lambda_{2}\right)(n+1)-2 n^{3}-6 n^{2}-6 n+8 \pi^{2} y^{2}-2\right) y \frac{\partial}{\partial y} \\
& +\left(\lambda_{1}+\lambda_{2}-6 n^{2}-18 n+4 \pi^{2} y^{2}-14\right) y^{2} \frac{\partial^{2}}{\partial y^{2}} \\
& -4(n+2) y^{3} \frac{\partial^{3}}{\partial y^{3}}-y^{4} \frac{\partial^{2}}{\partial y^{2}}, \\
L_{1}^{m}= & \frac{d}{d z_{1}}\left(\left(z_{1}^{2}-1\right) \frac{d}{d z_{1}}\right)-\frac{m^{2}}{z_{1}^{2}-1}, \\
L_{2}^{m}= & \frac{d}{d z_{2}}\left(\left(z_{2}^{2}-1\right) \frac{d}{d z_{2}}\right)-\frac{m^{2}}{z_{2}^{2}-1}, \\
L^{\prime}= & -\left(L_{1}^{0} L_{2}^{n}-n(n+1) L_{1}^{0}-n(n+1) L_{2}^{n}+n^{4}+2 n^{3}+n^{2}-8 \pi^{2} y^{2}\right) \\
& +2\left(\left(L_{1}^{0}+L_{2}^{n}\right)(n+1)-2 n^{3}-6 n^{2}-6 n+8 \pi^{2} y^{2}-2\right) y \frac{\partial}{\partial y}
\end{aligned}
$$




$$
\begin{aligned}
& +\left(L_{1}^{0}+L_{2}^{n}-6 n^{2}-18 n+4 \pi^{2} y^{2}-14\right) y^{2} \frac{\partial^{2}}{\partial y^{2}} \\
& -4(n+2) y^{3} \frac{\partial^{3}}{\partial y^{3}}-y^{4} \frac{\partial^{4}}{\partial y^{4}} .
\end{aligned}
$$

Then we have

$$
\begin{aligned}
& L \int_{0}^{\infty}\left(\int_{1}^{\infty} R_{\nu_{1}}^{0}\left(z_{1}\right) P_{\nu_{2}}^{n}\left(z_{2}\right)\left(-2 \pi y z_{2}\right)^{-n}\left(z_{2}^{2}-1\right)^{n / 2} e^{-2 \pi z_{1} z_{2} y} d z_{2}\right) d z_{1} \\
& =\int_{0}^{\infty} \int_{1}^{\infty}\left(-L_{1}^{0} R_{\nu_{1}}^{0}\left(z_{1}\right) L_{2}^{n} P_{\nu_{2}}^{n}\left(z_{2}\right)+n(n+1)\left(L_{1}^{0} R_{\nu_{1}}^{0}\left(z_{1}\right) P_{\nu_{2}}^{n}\left(z_{2}\right)\right.\right. \\
& \left.\quad+R_{\nu_{1}}^{0}\left(z_{1}\right) L_{2}^{n} P_{\nu_{2}}^{n}\left(z_{2}\right)\right)-\left(n^{4}+2 n^{3}+n^{2}-8 \pi^{2} y^{2}\right) R_{\nu_{1}}^{0}\left(z_{1}\right) P_{\nu_{2}}^{n}\left(z_{2}\right) \\
& \quad+2\left((n+1)\left(L_{1}^{0} R_{\nu_{1}}^{0}\left(z_{1}\right) P_{\nu_{2}}^{n}\left(z_{2}\right)+R_{\nu_{1}}^{0}\left(z_{1}\right) L_{2}^{n} P_{\nu_{2}}^{n}\left(z_{2}\right)\right)\right. \\
& \left.\quad+R_{\nu_{1}}^{0}\left(z_{1}\right) P_{\nu_{2}}^{n}\left(z_{2}\right)\left(-2 n^{3}-6 n^{2}-6 n+8 \pi^{2} y^{2}-2\right)\right) y \frac{\partial}{\partial y} \\
& \quad+\left(L_{1}^{0} R_{\nu_{1}}^{0}\left(z_{1}\right) P_{\nu_{2}}^{n}\left(z_{2}\right)+R_{\nu_{1}}^{0}\left(z_{1}\right) L_{2}^{n} P_{\nu_{2}}^{n}\left(z_{2}\right)\right. \\
& \left.\quad+R_{\nu_{1}}^{0}\left(z_{1}\right) P_{\nu_{2}}^{n}\left(z_{2}\right)\left(-6 n^{2}-18 n+4 \pi^{2} y^{2}-14\right)\right) y^{2} \frac{\partial^{2}}{\partial y^{2}}-R_{\nu_{1}}^{0}\left(z_{1}\right) P_{\nu_{2}}^{n}\left(z_{2}\right) \\
& \left.\quad \times\left(4(n+2) y^{3} \frac{\partial^{3}}{\partial y^{3}}+y^{4} \frac{\partial^{4}}{\partial y^{4}}\right)\right)\left(-2 \pi y z_{2}\right)^{-n}\left(z_{2}^{2}-1\right)^{n / 2} e^{-2 \pi z_{1} z_{2} y} d z_{1} d z_{2} \\
& =\int_{0}^{\infty} \int_{1}^{\infty} R_{\nu_{1}}^{0}\left(z_{1}\right) P_{\nu_{2}}^{n}\left(z_{2}\right) L^{\prime}\left(\left(-2 \pi y z_{2}\right)^{-n}\left(z_{2}^{2}-1\right)^{n / 2} e^{-2 \pi z_{1} z_{2} y}\right) d z_{1} d z_{2} \\
& \quad+n(n+1) \frac{\partial}{\partial z_{1}} R_{\nu_{1}}^{0}(0) \int_{1}^{\infty} P_{\nu_{2}}^{n}\left(z_{2}\right)\left(-2 \pi y z_{2}\right)^{-n}\left(z_{2}^{2}-1\right)^{n / 2} z_{2}^{-2} d z_{2}
\end{aligned}
$$

by partial integration. We note that all integrals should be considered as limits of integrals of finite interval. Since we easily see that

$$
\begin{aligned}
& L^{\prime}\left(\left(-2 \pi y z_{2}\right)^{-n}\left(z_{2}^{2}-1\right)^{n / 2} e^{-2 \pi z_{1} z_{2} y}\right)=0 \\
& L y^{-m}=\frac{1}{64}\left(\lambda_{1}-(n-m)^{2}-(n-m)\right)\left(\lambda_{2}-(n-m)^{2}-(n-m)\right) y^{-m} \\
& \quad-\frac{1}{16} \pi^{2}(n-1)(n-2) y^{-m+2},
\end{aligned}
$$

there exists a polynomial $h_{1}$ in $y^{-1}$ of degree $n$ such that $A_{n}$ is a solution of $(1,14)$. In the same way we can show existence of $h_{2}, h_{3}, h_{4}$ such that $B_{n}, C_{n}, D_{n}$ are solutions of (1.14). It is easy to see that $y A_{n}, y B_{n}$ are bounded when $y \rightarrow \infty$ and that $C_{n}$ and $D_{n}$ are rapidly decreasing and increasing respectively when $y \rightarrow \infty$. Since we easily see that $A_{n}$, $B_{n}$ have different asymptotic expansions by considering 


$$
\begin{aligned}
& \int_{0}^{\epsilon}\left(\int_{1}^{\infty} R_{\nu_{1}}^{0}\left(z_{1}\right) P_{\nu_{2}}^{n}\left(z_{2}\right)\left(-2 \pi y z_{2}\right)^{-n}\left(z_{2}^{2}-1\right)^{n / 2} e^{-2 \pi z_{1} z_{2}} d z_{2}\right) d z_{1} \\
& \int_{0}^{\epsilon}\left(\int_{1}^{\infty} S_{\nu_{1}}^{0}\left(z_{1}\right) P_{\nu_{2}}^{n}\left(z_{2}\right)\left(-2 \pi y z_{2}\right)^{-n}\left(z_{2}^{2}-1\right)^{n / 2} e^{-2 \pi z_{1} z_{2}} d z_{2}\right) d z_{1}
\end{aligned}
$$

for small $\varepsilon$, functions $A_{n}, B_{n}, C_{n}, D_{n}$ are linearly independent.

By the recurrence relation (1.9.1) and the partial integration using (1.17), we especially obtain

Proposition 2. The functions of $x, y$

$$
\begin{aligned}
C_{n}(x, y)= & \left(x^{2}-y^{2}\right) \int_{1}^{\infty} \int_{1}^{\infty} P_{\nu_{1}}^{n}\left(z_{1}\right) P_{\nu_{2}}^{n}\left(z_{2}\right) \\
& \times J_{n}\left(2 \pi i\left(z_{1}^{2}-1\right)^{1 / 2}\left(z_{2}^{2}-1\right)^{1 / 2} x\right) e^{-2 \pi z_{1} z_{2} y} d z_{1} d z_{2}
\end{aligned}
$$

are solutions of the equations (1.8.1), (1.8.2) where $J_{n}$ denotes the Bessel function of the first kind.

THEOREM 1. Put $\nu_{1}=\frac{-1+\sqrt{1+4 \lambda_{1}}}{2}, \quad \nu_{2}=\frac{-1+\sqrt{1+4 \lambda_{2}}}{2}$, and assume that $-1<\Re e \nu_{1}<0,-1<\Re e \nu_{2}<0$ and that $\lambda_{1}, \lambda_{2} \in \mathbb{R}$ are not integers.

Let $f(X+i Y)=g(Y) e^{2 \pi i \operatorname{tr} X}$ be a generalized Whittaker functions i.e., $f(X+i Y)$ satisfies $\Delta_{1} f=d_{1} f, \Delta_{2} f=d_{2} f$ with $d_{1}, d_{2}$ in (1.13). Assume that $g(Y)$ is a real analytic function of $t_{1}-t_{2}$ with

$$
Y=\left(\begin{array}{ll}
y_{1} & y_{2} \\
y_{2} & y_{3}
\end{array}\right)=\left(\begin{array}{ll}
t_{1} & 0 \\
0 & t_{2}
\end{array}\right)\left[\begin{array}{rr}
\cos \theta & -\sin \theta \\
\sin \theta & \cos \theta
\end{array}\right]
$$

and that for all positive integers $m, a, b, c$, the convergence

$$
(\operatorname{tr} Y)^{m} \frac{\partial^{a+b+c}}{\partial y_{1}^{a} \partial_{2}^{b} \partial y_{3}^{c}} g(Y) \rightarrow 0
$$

holds uniformly on compact sets of $y_{1}-y_{3}$ and $y_{2}$ when $\operatorname{tr} Y \rightarrow 0$.

Then $\mathrm{g}(Y)$ is expanded as follows:

$$
g(Y)=\sum_{n \in Z} b_{n} C_{n}\left(t_{1}+t_{2}, t_{1}-t_{2}\right) e^{2 n i 0}
$$

where $C_{n}(x, y)$ is the function defined in Proposition 2 and $b_{n} \in \mathbb{C}$.

Proof. Put $x=t_{1}-t_{2}, y=t_{1}+t_{2}$. Then

$$
\begin{aligned}
\left.\frac{\partial}{\partial x^{n}}\right|_{x=0} \int_{0}^{\pi} g(Y) e^{-2 i n \theta} d \theta & =\int_{0}^{\pi}\left(\frac{\cos 2 \theta}{2}\left(\frac{\partial}{\partial y_{1}}-\frac{\partial}{\partial y_{3}}\right)\right. \\
& \left.-\frac{\sin 2 \theta}{2} \frac{\partial}{\partial y_{2}}\right)\left.^{n}\right|_{y_{1}-y_{3}=0, y_{2}=0} g(Y) e^{-2 i n \theta} d \theta
\end{aligned}
$$


holds. Since the left hand side of (1.26) is a solution of (1.14), it should be one of the functions in Proposition 1. The right hand side of (1.26) decreases rapidly by the assumption and therefore it equals to $C_{n}(y)$ in Proposition 1.

\section{$\S 2$.}

Here we introduce a certain theta function and show the commutation relations of invariant differential operators and explain their connection with the generalized Whittaker functions. We deal with $L$-function of biquadratic fields with Grössencharacter at the end.

For $\left(g_{2}, g_{2}\right) \in S L_{2}(\mathbb{R}) \times S L_{2}(\mathbb{R})$, put

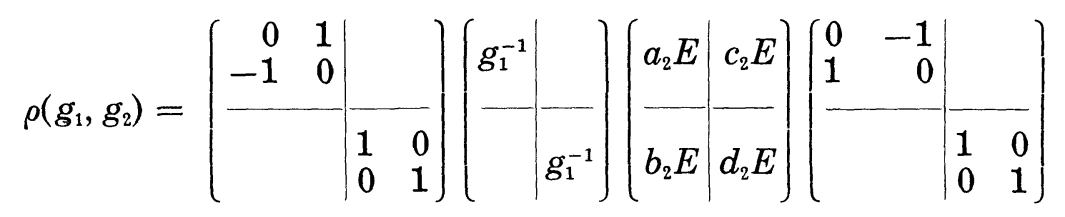

with $E=\left(\begin{array}{ll}1 & 0 \\ 0 & 1\end{array}\right), g_{2}=\left(\begin{array}{ll}a_{2} & b_{2} \\ c_{2} & d_{2}\end{array}\right)$. Then $\rho\left(g_{1}, g_{2}\right)$ is an element in the orthogonal group for $\left(\begin{array}{cc}0 & E \\ E & 0\end{array}\right)$. For an odd squarefree integer $N$, we define a lattice $\mathscr{L}$ by

$$
\mathscr{L}=\left(\left\{\begin{array}{ll}
m_{11} & m_{12} \\
m_{21} & m_{22} \\
m_{31} & m_{32} \\
m_{41} & m_{42}
\end{array}\right) \mid m_{i j} \in \mathbb{Z} \text { for }(i, j) \neq(1,2), N m_{12} \in \mathbb{Z}\right\} .
$$

For $Z=X+i Y \in H_{2}$ and a Dirichlet character $\chi$ modulo $N$, put

$$
\begin{aligned}
\Theta_{x}\left(Z,\left(g_{1}, g_{2}\right)\right)= & |Y| \quad \sum_{m=\left(\begin{array}{cc}
* & m_{12} \\
* & * \\
* & *
\end{array}\right) \in \mathscr{C}} \chi\left(N m_{12}\right) \\
& \times \exp \left(\pi i N \operatorname{tr}\left(X\left(\left(\begin{array}{cc}
0 & E \\
E & 0
\end{array}\right)\left[\rho\left(g_{1}, g_{2}\right) m\right]\right)\right)\right) \\
& \times \exp \left(-\pi N \operatorname{tr}\left(Y\left(\left(\begin{array}{cc}
E & 0 \\
0 & E
\end{array}\right)\left[\rho\left(g_{1}, g_{2}\right) m\right]\right)\right)\right)
\end{aligned}
$$

where $S[T]$ means ${ }^{t} T S T$. The function in (2.3) is usually called theta function. Put $g_{x+i y}=\left(\begin{array}{cc}1 & x \\ 0 & 1\end{array}\right)\left(\begin{array}{cc}y^{1 / 2} & 0 \\ 0 & y^{-1 / 2}\end{array}\right)$ for $x+i y \in H_{1}$, the upper half plane, and put

$$
\theta\left(Z, z_{1}, z_{2}\right)=\Theta_{x}\left(Z,\left(g_{z_{1}}, g_{z_{3}}\right)\right) .
$$

Then using Nakajima [10] where the generators of the center $\mathscr{C}$ of the 
universal enveloping algebra of $\mathfrak{s p}(2, \mathbb{R})$ are given, we have

ThEOREM 2.1) For $z_{1}=x_{1}+i y_{1}, z_{2}=x_{2}+i y_{2} \in H_{1}$,

$$
\begin{aligned}
\Delta_{1} \theta\left(Z, z_{1}, z_{2}\right) & =\frac{1}{8}\left(y_{1}^{2}\left(\frac{\partial^{2}}{\partial x_{1}^{2}}+\frac{\partial^{2}}{\partial y_{1}^{2}}\right)+y_{2}^{2}\left(\frac{\partial^{2}}{\partial x_{2}^{2}}+\frac{\partial^{2}}{\partial y_{2}^{2}}\right)-2\right) \theta\left(Z, z_{1}, z_{2}\right), \\
\Delta_{2} \theta\left(Z, z_{1}, z_{2}\right) & =\left(\frac{1}{256}\left(y_{1}^{2}\left(\frac{\partial^{2}}{\partial x_{1}^{2}}+\frac{\partial^{2}}{\partial y_{1}^{2}}\right)-y_{2}^{2}\left(\frac{\partial^{2}}{\partial x_{2}^{2}}+\frac{\partial^{2}}{\partial y_{2}^{2}}\right)\right)^{2}\right. \\
& \left.-\frac{1}{32}\left(y_{1}^{2}\left(\frac{\partial^{2}}{\partial x_{1}^{2}}+\frac{\partial^{2}}{\partial y_{1}^{2}}\right)+y_{2}^{2}\left(\frac{\partial^{2}}{\partial x_{2}^{2}}+\frac{\partial^{2}}{\partial y_{2}^{2}}\right)\right)+\frac{3}{64}\right) \theta\left(Z, z_{1}, z_{2}\right) .
\end{aligned}
$$

Note that $\Delta_{1}, \Delta_{2}$ defined in $\S 1$ are differential operators with respect to $Z$ not to $z_{1}, z_{2}$.

Proof. Put $V=M_{2,2}(\mathbb{R})$ and define the Weil representation $r$ of $G=S p(2, \mathbb{R})$ on $\mathscr{S}(V \times V)$ by

$$
\begin{aligned}
& r\left(\left(\begin{array}{cc}
E & X \\
0 & E
\end{array}\right)\right) f\left(\begin{array}{l}
X_{1} \\
X_{2}
\end{array}\right)=\exp \left(2 \pi i \operatorname{tr}\left(X^{t} X_{1} X_{2}\right)\right) f\left(\begin{array}{l}
X_{1} \\
X_{2}
\end{array}\right) \\
& r\left(\left(\begin{array}{cc}
A & 0 \\
0 & { }^{t} A^{-1}
\end{array}\right)\right) f\left(\begin{array}{l}
X_{1} \\
X_{2}
\end{array}\right)=\operatorname{det}(A) f\left(\begin{array}{l}
X_{1} A \\
X_{2} A
\end{array}\right) \\
& r\left(\left(\begin{array}{cc}
0 & E \\
-E & 0
\end{array}\right)\right) f\left(\begin{array}{l}
X_{1} \\
X_{2}
\end{array}\right)=\int_{V} \int_{V} f\left(\begin{array}{l}
Y_{1} \\
Y_{2}
\end{array}\right) \exp \left(2 \pi i \operatorname{tr}\left({ }^{t} Y_{1} X_{2}+{ }^{t} Y_{2} X_{1}\right)\right) d Y_{1} d Y_{2}
\end{aligned}
$$

for $f \in \mathscr{S}(V \times V)$ where $X_{1}, X_{2} \in V$ and $d\left(\begin{array}{ll}x_{11} & x_{12} \\ x_{21} & x_{22}\end{array}\right)=d x_{11} d x_{12} d x_{21} d x_{22}$. The representation $r$ of $G$ derives the representation $\tilde{r}$ of $\mathscr{C}$. Let $\Lambda_{1}, \Lambda_{2}$ be generators of $\mathscr{C}$ in [10]. Then we can express $\tilde{r}\left(\Lambda_{1}\right), \tilde{r}\left(\Lambda_{2}\right)$ by differential operators $\frac{\partial}{\partial x_{11}}, \cdots, \frac{\partial}{\partial x_{42}}$ for

$$
f\left(\begin{array}{ll}
x_{12} & x_{12} \\
x_{21} & x_{22} \\
x_{31} & x_{32} \\
x_{41} & x_{42}
\end{array}\right) \in \mathscr{S}(V \times V)
$$

$\rho\left(g_{1}, g_{2}\right)$ acts on $\mathscr{S}(V \times V)$ by

$$
\left(\rho\left(g_{1}, g_{2}\right) f\right)\left(\begin{array}{l}
X_{1} \\
X_{2}
\end{array}\right)=f\left(\rho\left(g_{1}, g_{2}\right)\left(\begin{array}{l}
X_{1} \\
X_{2}
\end{array}\right)\right)
$$

$\rho$ derives the representation $\tilde{\rho}$ of the direct product of two copies of the center of the universal enveloping algebra $c$ of $\mathfrak{z}(2, \mathbb{R})$ which is generated by the Casimir operator $\lambda$. $\tilde{\rho}(\lambda, 1), \tilde{\rho}(1, \lambda)$ also can be expressed by $\frac{\partial}{\partial x_{11}}, \cdots, \frac{\partial}{\partial x_{42}}$.

\footnotetext{
1) A computer machine is used to prove this theorem.
} 
Comparing these expressions for $\tilde{r}\left(\Lambda_{1}\right), \tilde{r}\left(\Lambda_{2}\right), \tilde{\rho}(\lambda, 1), \tilde{\rho}(1, \lambda)$ by a computer machine, we obtain desired results.

Let $\varphi_{1}, \varphi_{2}$ be Maass wave cusp forms with the character $\chi$ satisfying $y^{2}\left(\frac{\partial^{2}}{\partial x^{2}}+\frac{\partial^{2}}{\partial y^{2}}\right) \varphi_{1}(z)=\lambda_{1} \varphi_{1}(z), y^{2}\left(\frac{\partial^{2}}{\partial x^{2}}+\frac{\partial^{2}}{\partial y^{2}}\right) \varphi_{2}(z)=\lambda_{2} \varphi_{2}(z) . \quad$ Put $d_{0} z=$ $y^{-2} d x d y$ for $z=x+i y$, and define

$$
F_{\varphi_{1}, \varphi_{2}}(Z)=\int_{\Gamma \backslash H_{1}} \int_{\Gamma \backslash H_{1}} \theta\left(Z, z_{1}, z_{2}\right) \varphi_{1}\left(z_{1}\right) \varphi_{2}\left(z_{2}\right) d_{0} z_{1} d_{0} z_{2}
$$

with $\Gamma=\Gamma_{0}(N)$. Then we have

$$
\Delta_{1} F_{\varphi_{1}, \varphi_{2}}(Z)=d_{1} F_{\varphi_{1}, \varphi_{2}}(Z), \quad \Delta_{2} F_{\varphi_{1}, \varphi_{2}}(Z)=d_{2} F_{\varphi_{1}, \varphi_{2}}(Z),
$$

with $d_{1}, d_{2}$ defined by the equalities (1.13) for $\lambda_{1}, \lambda_{2}$, and

$$
F_{\varphi_{1}, \varphi_{2}}(\sigma Z)=\chi(d) F_{\varphi_{1}, \varphi_{2}}(Z)
$$

holds for $\sigma=\left(\begin{array}{cc|cc}* & * & * & * \\ * & * & * & * \\ \hline * & * & a & b \\ * & * & c & d\end{array}\right) \in \Gamma_{2}$ where

$$
\Gamma_{2}=\left\{\sigma=\left(\begin{array}{llll}
a_{11} & a_{12} & a_{13} & a_{14} \\
a_{21} & a_{22} & a_{23} & a_{24} \\
a_{31} & a_{32} & a_{33} & a_{34} \\
a_{41} & a_{42} & a_{43} & a_{44}
\end{array}\right) \begin{array}{l}
\sigma \in S p(2, \mathbb{Q}), \\
a_{21}, a_{31}, a_{32}, a_{41}, a_{42} \in N \mathbb{Z}, \\
N a_{13} \in \mathbb{Z}, \text { other } a_{i j} \in \mathbb{Z}
\end{array}\right\}
$$

There exists a lattice $\mathscr{T}$ in $\left\{\left(\begin{array}{ll}a & b \\ c & d\end{array}\right) \mid a, b, c \in \mathbb{Q}\right\}$ containing $\left(\begin{array}{ll}N & 0 \\ 0 & 1\end{array}\right)$ such that $F_{\varphi_{1}, \varphi_{2}}(Z)$ is expanded as follows:

$$
F_{\varphi_{1}, \varphi_{2}}(Z)=\sum_{T \in \mathscr{T}} A(T, Y) e^{2 \pi i \operatorname{tr}(T X)} .
$$

Hence

$$
W(Y)=A\left(\left(\begin{array}{cc}
N & 0 \\
0 & 1
\end{array}\right),\left(\begin{array}{cc}
\sqrt{\bar{N}^{-1}} & 0 \\
0 & 1
\end{array}\right) Y\left(\begin{array}{cc}
\sqrt{\bar{N}^{-1}} & 0 \\
0 & 1
\end{array}\right)\right)
$$

is a generalized Whittaker function. A direct calculation shows that $W\left(\left(\begin{array}{ll}t_{1} & 0 \\ 0 & t_{2}\end{array}\right)\left[\begin{array}{rr}\cos \theta & -\sin \theta \\ \sin \theta & \cos \theta\end{array}\right]\right)$ is equal to $\sum_{n \in Z} b_{n} C_{n}\left(t_{1}-t_{2}, t_{1}+t_{2}\right) e^{n i \theta}$ and $b_{0}$ is equal to

$$
\sum_{j=1}^{h} \chi\left(a_{j}\right) \varphi_{1}\left(\frac{-a_{j}}{c_{j} N+\sqrt{-N}}\right) \varphi_{2}\left(\frac{-a_{j}}{c_{j} N+\sqrt{-N}}\right)
$$


up to a constant multiple, where $\mathfrak{H}_{j}=a_{j} \mathbb{Z}+\left(c_{j} N+\sqrt{-N}\right) \mathbb{Z}$ runs over the full set of representatives of the jdeal class group of $\mathbb{Q}(\sqrt{-N})$ with class number $h$ and $C_{n}\left(t_{1}, t_{2}\right)$ is same as defined in Proposition 2.

Note that the corresponding $L$-function (the spinor $L$-function) of $F_{\varphi_{1}, \varphi_{2}}(Z)$ is the product $L\left(s, \varphi_{1}\right) L\left(s, \varphi_{2}\right)$ of $L$-functions of $\varphi_{1}$ and $\varphi_{2}$. Let $K_{i}=\mathbb{Q}\left(\sqrt{d_{i}}\right)$ be a real quadratic field with discriminant $d_{i}$ for $i=1,2,3$. Let $d_{3}=d_{1} d_{2}$ and assume the class number of $K=K_{3}$ is one. Let o be the ring of integers in $K$ and $E_{+}$the group of all totally positive units in o. Put

$$
\begin{aligned}
& g_{1}\left(z, \xi_{m}\right)=\sum_{\substack{\mu \in 0 / E_{+}+\\
\mu \neq 0}} \xi_{m}(\mu) y^{1 / 2} K_{i m \kappa}\left(2 \pi\left|N_{K / \mathrm{Q}} \mu\right| y\right) \cos \left(2 \pi x N_{K / \mathrm{Q}} \mu\right), \\
& g_{2}\left(z, \xi_{m}\right)=\sum_{\substack{\mu \in 0 / E_{+}+\\
\mu \neq 0}} \xi_{m}(\mu) \psi(\mu) y^{1 / 2} K_{i m \kappa}\left(2 \pi\left|N_{K / \mathrm{Q}} \mu\right| y\right) \cos \left(2 \pi x N_{K / \mathrm{Q}} \mu\right)
\end{aligned}
$$

where $\xi_{m}(\mu)=\left.|\mu| \mu^{\prime}\right|^{i m_{\kappa}}, \quad \kappa=2 \pi / \log \varepsilon, \psi(\mu)=\left(\frac{d_{1}}{N_{K / \mathrm{O}} \mu}\right), \varepsilon$ the fundamental unit in $K, K_{i m \kappa}$ the modified Bessel function. Then $g_{1}$ is a Maass wave form on $\Gamma_{0}\left(d_{1} d_{2}\right)$ with character $\left(\frac{d_{1} d_{2}}{*}\right)$ and $g_{2}$ a Maass wave form on $\Gamma_{0}\left(d_{1}^{2} d_{2}\right)$ with character $\left(\frac{d_{1} d_{2}}{*}\right)$. Though in this case $N=d_{1}^{2} d_{2}$ is not squarefree, we can define $\Theta, \theta$ and $F_{\varphi_{1}, \varphi_{2}}$ for $\varphi_{1}=g_{1}, \varphi_{2}=g_{2}$ in the same way as above. In this case $L$-function corresponding to $F_{\varphi_{1}, \varphi_{2}}$ is

$$
\sum_{a} \xi\left(N_{F / k} \mathfrak{a}\right) N_{F / \mathbb{Q}} \mathfrak{a}^{-s}
$$

where $\mathfrak{a}$ runs over all integral ideals in $F=\mathbb{Q}\left(\sqrt{d_{1}}, \sqrt{d_{2}}\right)$. (2.10) may be a little more complicated in this case.

Added in proof. The author would like to express his hearty thanks to Prof. T. Oda and Mr. A. Hori who corrected mistaks of sign in the relation between $\nu_{1}, \nu_{2}$ and $\lambda_{1}, \lambda_{2}$.

\section{REFERENCES}

[1] M. Eichler, Quadratische Formen und orthogonale Groupen, Springer.

[2] S. Friedberg, Differential operators and theta series, Trans. Amer. Math. Soc., 287 (1985), 569-589.

[ 3 ] G. Kaufhold, Dirichletsche Reihe mit Funktionalgleichung in der Theorie der Modulfunktion 2. Grades, Math. Ann., 137 (1959), 454-476.

[4] M. Koecher, Über Thetareihen indefiniter quadratischer Formen, Math. Nachr., 9 (1953), 51-85. 
[5] H. Maass, Die Differentialgleichungen in der Theorie der Siegelschen Modulfunktionen, Math. Ann., Bd., 126 (1953), 44-68.

[6] - U Über eine neue Art von nichtanalitischen automorphen Funktionen und die Bestimmung Dirichletscher Reihen durch Functionalgleichungen, Math. Ann., 121 (1949), 141-183.

[ 7 ] - Dirichletsche Reihen und Modulfunktionen zweiten Grades, Acta Arith., 24 (1973), 225-238.

[ 8 ] — Über die räumliche Verteilung der Punkte in Gittern mit indefiniter Metrik, Math. Ann., 138 (1959), 287-315.

[9] S. Nakajima, On invariant differential operators on bounded symmetric domains of type IV, Proc. Japan Acad., 58, Ser. A (1982), 235-238.

[10] - Invariant differential operators on $S O(2, q) / S O(2) \times S O(q)(q \geq 3)$, Master these.

[11] G. Shimura, Confluent hypergeometric functions on tube domains, Math. Ann., 265 (1982), 269-302.

[12] H. Yamashita, On Whittaker vectors for generalized Gelfand-Graev representations of semisimple Lie groups, J. Math. Kyoto Univ., 26-2 (1986), 263-298.

[13] - On Whittaker vectors for generalized Gelfand-Graev representations of semisimple Lie groups, Proc. Japan Acad., 61, Ser. A (1985), 213-216.

[14] - Finite multiplicity theorems for induced representations of semisimple Lie groups and their applications to generalized Gelfand-Graev representations, Proc. Japan Acad., 63, Ser. A (1987), 153-156.

[15] - Multiplicity one theorems for generalized Gelfand-Graev representations of semisimple Lie groups and Whittaker models for the discrete series, Preprint.

[16] H. Yoshida, Siegel's modular forms and the arithmetic of quadratic forms, Invent. Math., 60 (1980), 193-248.

[17] —-, On Siegel modular forms obtained from theta series, J. reine angew. Math., 352 (1984), 184-219.

Nagoya City College of

Child Education

Owariasahi, 488

Japan 